\title{
A Multi-Wavelength Access Network featuring WiMAX Transmission over GPON Links
}

\author{
M. Milosavljevic ${ }^{(1)}$, M. P. Thakur ${ }^{(2)}$, P. Kourtessis ${ }^{(1)}$, J. E. Mitchell ${ }^{(2)}$, J. M. Senior ${ }^{(1)}$ \\ (1) Optical Networks Group, STRI, Univ. of Hertfordshire, UK, m.milosavljevic@herts.ac.uk \\ ${ }^{(2)}$ Department of Electronic and Electrical Engineering, UCL, London, UK, m.thakur@ee.ucl.ac.uk
}

\begin{abstract}
An IEEE802.16e compliant PON architecture with wavelength band overlay has been demonstrated. Transparent transmission of mobile-WiMAX RF channels over multi-wavelength GPON links, based on FDM, has demonstrated at remote receivers standard WiMAX EVM figures with $1 E^{11}$ GPON bit-error-rates.
\end{abstract}

\section{Introduction}

Following the escalating demand of end-users for bandwidth, the last-mile infrastructure upgrade is imminent. Next generation access networks are also expected to provide greater scalability and mobility than the existing broadband channels. In that direction the convergence of optical topologies and the ubiquitous wireless medium can uniquely provide high bandwidth, low infrastructure deployment costs and network redundancy ${ }^{1}$.

In view of these impending requirements, a novel architectural platform has been demonstrated to implement the transparent transmission of WiMAX and GPON signals in conjunction with extended wavelength band overlay over legacy PONs. Frequency division multiplexing (FDM) was used to address individual base stations sharing a single wavelength to avoid interference with GPON while achieving high spectral efficiency.

\section{Network Architecture}

A comprehensive diagram of the proposed network architecture, incorporating the developed experimental setup, is shown in Fig. 1. The key feature of this expandable topology is the attainable dynamicity, through the application of extended wavelength band overlay $^{2}$. For each ONU/BS, the frequency shifted downstream WiMAX channels can be actively multiplexed on different wavelengths, therefore, relaxing the bandwidth requirements on the network components. The resulting

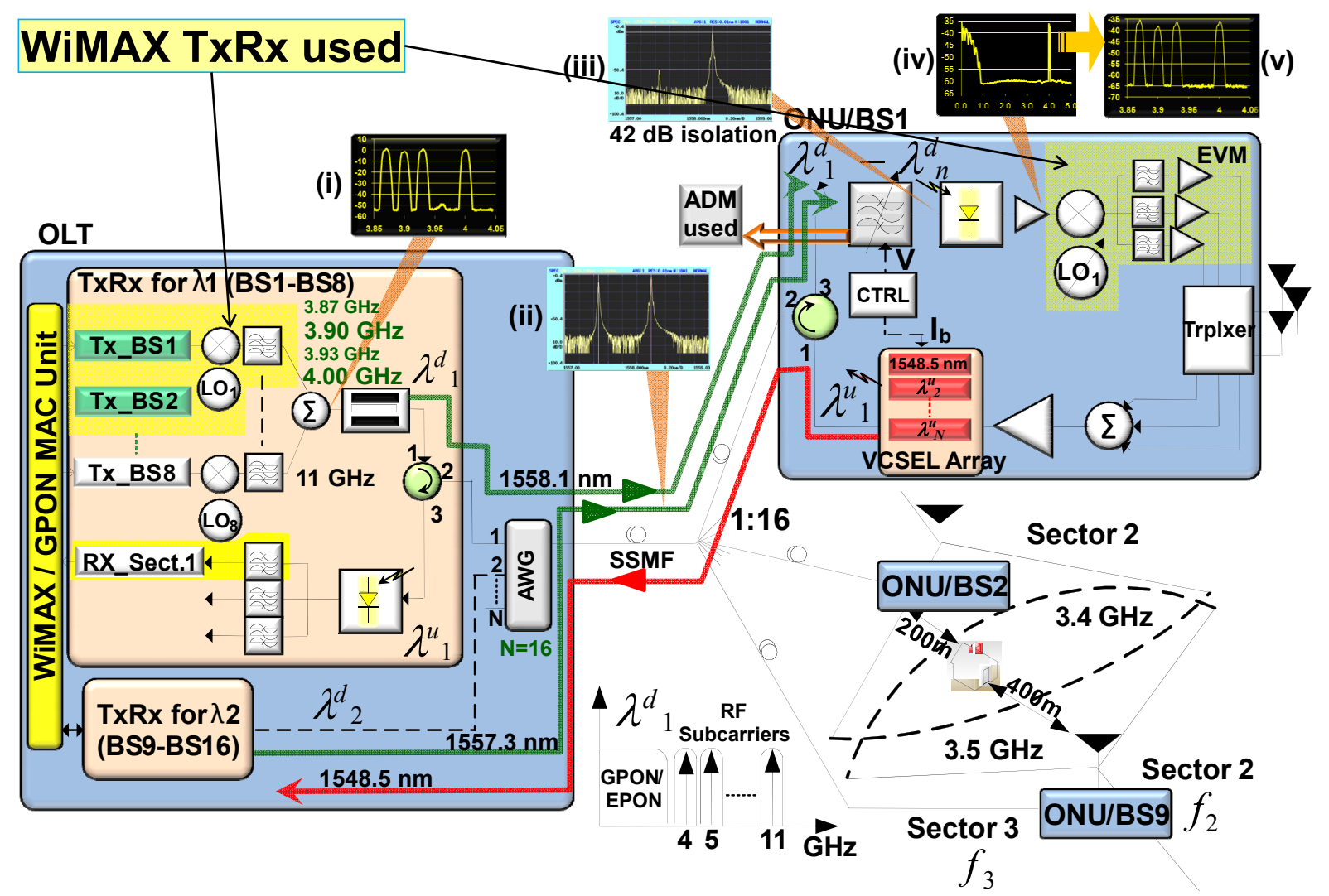

Fig. 1: WiMAX over multi-wavelength, power splitting PONs 
reduction in total FDM bandwidth minimizes the effects of fibre dispersion. This has been demonstrated in the network by the successful transmission of WiMAX channels over $40 \mathrm{~km}$ fibre without any dispersion compensation. In addition, any wavelength of the selected operating spectrum can be partly or exclusively assigned to different ONU/BSs, achieving service levels, similar to WDM-PONs. In hardware, this is implemented by the addition of a dense array waveguide grating (AWG) in the optical line terminal (OLT) and tuneable optical filters in ONU/BSs ${ }^{2}$. The centre frequency of the latter can be adjusted by the OLT by means of a control circuit.

Another significant feature of the proposed topology lays in the use of low-cost longwavelength VCSEL arrays $^{3}$ for upstream, to demonstrate colourless terminations with simple coupling optics, which are not limited by the non-linear effects of the optical fibre. Although RSOAs could possibly be investigated ${ }^{4}$, the Rayleigh backscattering is known to potentially degrade performance. VCSEL wavelength selection can be managed by implementing controls similar to tuning the ONU/BS filters. The VCSEL array approach in combination with TDMA $^{5}$, when multiple ONU/BSs are sharing a single wavelength, could also be applied.

\section{Experimental Setup}

To establish accurate signal routing to and from an ONU/BS, received error vector magnitudes (EVMs) for the WiMAX channels and bit error rates (BERs) for the GPON signals were investigated. Three IEEE802.16e channels, with the specification given in Tab. 1 , at $3.5 \mathrm{GHz}$ and $30 \mathrm{MHz}$ channel spacing were generated by transmitter for ONU/BS2 (Tx_BS2) using an Aeroflex PXI WiMAX transceiver. The fourth 3.5 GHz WiMAX channel was generated by Tx_BS2 for transmission measurements. The downstream WiMAX channel transmitter relative constellation error (RCE) was $-50 \mathrm{~dB}$ that is lower than the minimum standards ${ }^{6}$ requirement for 64-QAM modulation. This was due to the low RF powers fed to drive the Mach-Zehnder modulator (MZM).

Subsequently, the WiMAX channels were frequency shifted to $3.9 \mathrm{GHz}$ (3 channels) and 4 $\mathrm{GHz}$ (measurement channel) spectrum to address individual ONU/BSs. The four frequency shifted WiMAX channels are displayed as inset (i) in Fig. 1. The combined WiMax channels were then used to externally modulate the optical carrier. Having added the various optical component losses, including 7.75 $\mathrm{dB}$ for the $\mathrm{MZM}, 1 \mathrm{~dB}$ for the polarisation controller and $3.76 \mathrm{~dB}$ for the AWG; +0.9 dBm optical power was launched into the fibre. To demonstrate the fixed to mobile convergence, a baseband GPON channel at $1.25 \mathrm{Gbit} / \mathrm{s}$ was introduced. The combined spectrum was transmitted on $\lambda d 1=1558.1 \mathrm{~nm}$, through a $16 \times 1$ AWG to an ONU/BS of the corresponding PON, using various lengths of standard single-mode fibre (SSMF).

Tab. 1: IEE802.16e WiMAX transceivers parameters

\begin{tabular}{|c|c|c|}
\hline & Downstream & Upstream \\
\hline FFT size & \multicolumn{2}{|c|}{$\mathrm{FFT}=1024$} \\
\hline Modulation & $64-\mathrm{QAM}$ & $16-\mathrm{QAM}$ \\
\hline Coding & $2 / 3$ & $3 / 4$ \\
\hline BW & \multicolumn{2}{|c|}{$10 \mathrm{MHz}$} \\
\hline RCE & $-50 \mathrm{~dB}$ & $-25.8 \mathrm{~dB}$ \\
\hline Data rate & $25.2 \mathrm{Mbps}$ & $18.9 \mathrm{Mbps}$ \\
\hline
\end{tabular}

An additional un-modulated wavelength at $1557.3 \mathrm{~nm}$ was employed to accommodate interference at the ONU/BS. The resulting optical spectrum is given in Fig. 1 as inset (ii). To account for various splitter loses an optical attenuator was used after the fibre. At the ONU/BS, $50 \mathrm{GHz}$ optical band pass filter, was utilised prior to PIN detection with signal to interference ratio of $42 \mathrm{~dB}$ (inset (iii) in Fig. 1). The resulting WiMAX electrical spectrum was then down shifted in frequency to get the original WiMAX channels.

A single IEEE802.16e, $3.5 \mathrm{GHz}$ channel was generated upstream to directly modulate a VCSEL biased at $8.3 \mathrm{~mA}, \lambda \mathrm{u}^{1}=1548.5 \mathrm{~nm}$ and output power level of $-0.94 \mathrm{dBm}$ prior to being transmitted over the SSMF. The upstream WiMAX transmission assumes a noiseless radio channel and therefore the RCE at the ONU/BS1 was $-25.8 \mathrm{~dB}$. Also is this the same as RCE defined earlier. The optical signal was then routed through the corresponding AWG output port to the destination receivers (Rx_Sect.X) in the OLT.

\section{Results and Discussions}

For the received GPON signal, shown as inset (iv) in Fig. 1, transmitted over 23.2km fibre, BER of $1 \times 10^{-11}$ was noted, with the fixed optical received power, demonstrating error-free transmission for the wired users. For WiMAX channels, as the nonlinear optical modulator could significantly degrade network performance, EVM characteristics as a function of the MZM RF drive power in the OLT downstream were measured. 


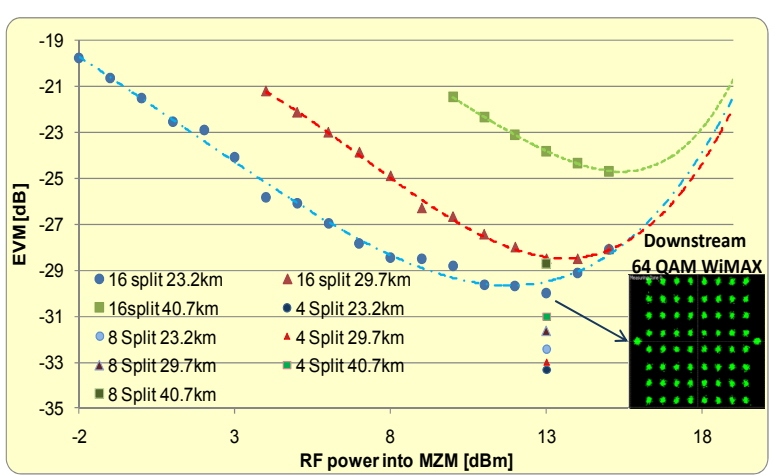

Fig. 2: Obtained EVM for $3.5 \mathrm{GHz}$ WiMAX channel at remote antenna downstream

As illustrated in Fig. 2, at low RF drive powers the signal is mainly impaired by noise, while at high powers, EVMs are dominated by the nonlinearity of the modulator. However, an EVM figure of higher than $-29 \mathrm{~dB}$ for $23.2 \mathrm{~km}$ fibre was achieved with $+13 \mathrm{dBm}$ RF fed to the MZM, matching the WiMAX requirements ${ }^{6}$ for 64-QAM $2 / 3$. The measurements were repeated for fibre lengths of $29.7 \mathrm{~km}$ and $40.7 \mathrm{~km}$. As displayed in Fig. 3, for lower split ratios, EVM below $-29 \mathrm{~dB}$ can be achieved for all fibre lengths without any dispersion compensation techniques. Therefore, the power penalty on longer fibres can be compensated by increasing the launch power.

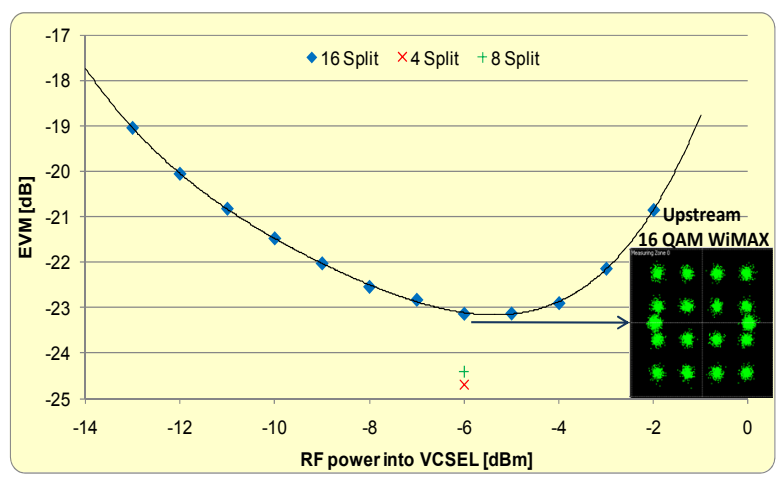

Fig. 3: Obtained EVM at the WiMAX OLT receiver for $3.5 \mathrm{GHz}$ channel

The upstream EVM at the OLT receiver against the RF power into the VCSEL is shown in Fig. 3. EVM of $-23 \mathrm{~dB}$ was noted for $23.2 \mathrm{~km}$ fibre and 16 split, matching the WiMAX standard ${ }^{6}$ for $16-$ QAM 3/4 modulation. The interaction of VCSEL laser chirp and fibre dispersion in analog optical modulation upstream had negligible effect on the received WiMAX channels in the OLT. Longer fibre lengths were not considered due to the VCSEL output power limitation. Similarly for downstream, at 4 and 8 splits, corresponding to higher received optical power, an EVM below $24 \mathrm{~dB}$ was measured. This demonstrates that higher output power VCSEL arrays ${ }^{3}$ can significantly reduce the EVM penalties. Hence, it can be concluded, that the proposed optical network provides transparent channel for standard wireless signal formats transmitted over multi-wavelength power-splitting.

Consequently, modelling results for the WiMAX downstream multipath transmission demonstrated a maximum BER of $10^{-4}$ without any channel coding, to represent the worst-case scenario, over $400 \mathrm{~m}$ overlapping cell circumference.

Finally, the constellation diagrams obtained at the WiMAX receivers after the phase and amplitude corrections are displayed in Fig. 2 and Fig. 3 as insets. The two outermost points on the constellations represent the pilot tones used for estimation purposes.

\section{Conclusions}

A highly-scalable network topology is described featuring transparent WiMAX and error-free GPON transmission by means of FDM over multi-wavelength power-splitting, using low-cost long-wavelength VCSEL arrays in ONU/BS. IEEE802.16e WiMAX standards compliance was demonstrated with EVM figures below -29 and $-23 \mathrm{~dB}$ for 25.2 and $18.9 \mathrm{Mbps}$, down/upstream respectively. $40 \mathrm{~km}$ downstream transmission without any dispersion compensation was readily achieved.

\section{Acknowledgments}

This work was supported by the European Network of Excellence, BONE project and ACCORDANCE, through the 7th ICT Framework Programme.

\section{References}

1 J. I. Kani et al., IEEE Comm. Mag., 47, 43, (2009).

2 G.984.5, ITU-T (2007).

3 W. Hofmann et al., Photon. Technol. Lett. 20, 291, (2008).

4 T. Jayasinghe et al., J. Opt. Netw. 6, 1025, (2007).

5 M. Milosavljevic et al., J. Opt. Netw. 8, 285, (2009).

6 IEEE Std 802.16 (2005) 